\title{
Design of Powered Floor Systems for Mobile Robots with Differential Evolution
}

\author{
Eric Medvet, Stefano Seriani, Alberto Bartoli, and Paolo Gallina \\ Department of Engineering and Architecture, University of Trieste, Trieste, Italy
}

\begin{abstract}
Mobile robots depend on power for performing their task. Powered floor systems, i.e., surfaces with conductive strips alternatively connected to the two poles of a power source, are a practical and effective way for supplying power to robots without interruptions, by means of sliding contacts. Deciding where to place the sliding contacts so as to guarantee that a robot is actually powered irrespective of its position and orientation is a difficult task. We here propose a solution based on Differential Evolution: we formally define problem-specific constraints and objectives and we use them for driving the evolutionary search. We validate experimentally our proposed solution by applying it to three real robots and by studying the impact of the main problem parameters on the effectiveness of the evolved designs for the sliding contacts. The experimental results suggest that our solution may be useful in practice for assisting the design of powered floor systems.
\end{abstract}

Keywords: Multi-objective optimization, Automatic design, Swarm robotics, Evolutionary robotics

\section{Introduction and related work}

Mobile robots are playing a role of increasing importance in current society. They are and will be aiding humans in performing tasks which may be dangerous, fatiguing, or boring due to repetitiveness. Examples range from persons transportation and goods delivering to surveillance.

One significant challenge in the field of mobile robots is how to provide power to the robots. The most common solution is to use batteries, which have, however, the apparent limitation that have to be recharged, causing a stop in the robot operations. A viable alternative consists in delivering power to the robot by electrifying the environment where it moves: this solution builds on the longestablished experience on supplying powers to mobile machines, the most notable examples being in transportation systems, e.g., trains [1] and trams [2]. Another large family of solutions is based on wireless power delivery, often realized by means of resonating coils $[3,4]$.

In this paper, we focus on powered floors: the surface on which the robot moves is covered with conductive strips, interleaved by narrow non-conductive strips, alternatively connected to positive and negative poles of a power source; 
the robots receive the power from the strips using a number of sliding contacts positioned on the bottom of their bodies. This solution fits well the scenario of lab experimentation with Swarm Robotics [5] or Evolutionary Robotics [6]. In this settings, small prototypical robots have to run for long time, without obstacles to their movements (as, e.g., wires for power supply), in a physically constrained environment which has to be easily observable and accessible to researcher, for experiment "debugging" purposes. The latter condition may be not met if, e.g., robots get the power from the floor and the ceiling. Indeed, powered floors have already been used in similar settings [7] and also inspired the design of robotic platforms tailored purposely to experimentation [8].

Designing a working powered floor system (i.e., robots and floor) requires to decide the width of the strips and the positions of the sliding contacts on the robot. While the first task is not subjected to many constraints, for the positions of the contacts one has to take in to account the shape of the robot and the presence of moving parts, parts which should not be covered (e.g., sensors), or parts which are too far or too close to the floor. Moreover, the fundamental requirement is that the contacts should be positioned in a way that guarantees that at least one contact is on a positive strip and one on a negative strip for any rotation and position of the robot. A fixed contacts design which aims at satisfying this condition, consisting of four contacts, has been already proposed in the 50 s by Claude Shannon [9, p. 678], but it might be unsuitable for some robots due to the constraints described above.

We here propose a method, based on Differential Evolution (DE) [10], for finding automatically the positions of the sliding contacts, given a description of the region of the robot which is suitable for hosting them, which guarantee that the robot can receive power from the floor in any rotation and position. DE is an Evolutionary Algorithm (EA) for continuous optimization which can be used for solving single- or multi-objective problems. We formally define the condition for the robot to be always powered, given the positions of its contacts, and we introduce, based on this definition, three quantitative objectives suitable for driving the search with DE. We experimentally assess the ability of our proposed solution to find the contact positions for three real robotic platforms (Thymio II, mBot, and Elisa-3) and investigate about which combination of the objectives is more effective and efficient in solving this task. Then, we explore the design space of the powered floor system problem: we focus on the two most relevant problem parameters, the width of the conductive strips and the maximum number of sliding contacts. The experimental evaluation suggests that our proposed solution based on DE is capable of designing automatically the salient part of a powered floor system, i.e., the positions of the contacts, in many different settings. The experiments also show that the quantitative objectives that we adopted for driving the search are indeed effective. 


\section{Scenario: mobile robots on powered floor}

We consider the scenario in which a mobile robotic platform moves on a powered floor.

The powered floor is composed of strips of conductive material interleaved by strips of non-conductive, insulating material (see Figure 1b). The conductive strips are alternatively connected to the positive and negative poles of a constant power source. For the purpose of this work and without loss of generality we assume that the strips length is infinite, there are infinite strips, all the conductive strips have the same width, and all the non-conductive strips have the same width.

The mobile robotic platform (the robot) is equipped with an array of sliding contacts, whose positions are fixed with respect to the geometry of the robot. For the purpose of this work we assume that each of the contacts is always in physical contact with the floor and is point-like (i.e., its size is zero along every axis). The robot may move, by translating and rotating, on the powered floor without any constraint.

The robot actually gets the power from the powered floor if there is at least one contact on a positive conductive strip and at least one contact on a negative conductive strip: if this condition is met, we say that the robot is in a powered condition and we call the contacts which enable this condition the powered contacts. We remark that there are no constraints on which contact is on which polarity: a simple rectifier circuit can be used to obtain a constant power source out of the powered contacts. The design of that circuit is orthogonal to this work.

The goal of the present study is to propose a method that, given (a) a maximum number $n$ of sliding contacts and (b) a description of a feasible region for the position of the sliding contacts with respect to the geometry of the robot, finds a design of the array of up to $n$ sliding contacts which (a) are in the feasible region and (b) allow the robot to be in a powered condition for any possible position, i.e., any possible combination of translation and rotation. We call a powered array an array of contacts which satisfies the two previous conditions, i.e., an array which guarantees that the robot is always in the powered condition.

\section{$2.1 \quad$ Formalization}

Without loss of generality, we assume that the strips are (all) parallel with the $y$-axis. This implies that the only relevant dimension is along the $x$-axis.

We denote by $w$ the width of the conductive strips and by $v$ the width of the non-conductive strips. We assume that the first strip starts at $x=0$, ends at $x=w$, and is connected to the positive pole.

We denote by $x$ the position of the a reference point of the robot, by $\omega$ the rotation of the robot, and by $A=\left\{\left(r_{1}, \phi_{1}\right), \ldots,\left(r_{n}, \phi_{n}\right)\right\}$ the array, i.e., the list of the $n$ positions $\left(r_{i}, \phi_{i}\right)$, expressed in polar coordinates, of each $i$ th contact with respect to the reference point of the robot. Figure 1a shows a schematic representation of the considered scenario and the corresponding notation; Figure $1 \mathrm{~b}$ shows a prototype of the powered floor and a robot. 


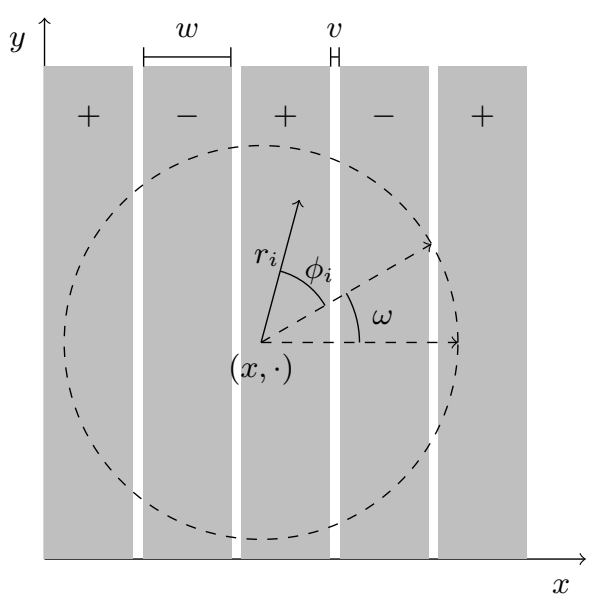

(a) Schematic representation.

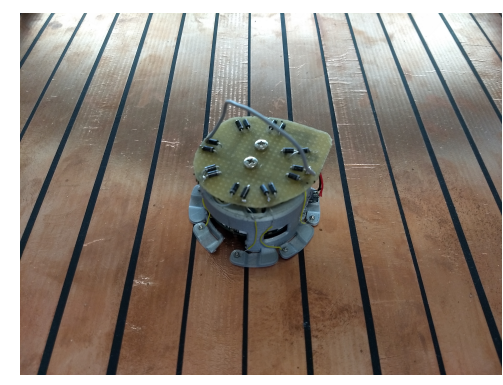

(b) Prototype.

Fig. 1. A schematic representation of the considered scenario (1a) and the picture of a prototype of the system (1b). In the former, the conductive strips are colored in gray, whereas the non-conductive strips are not colored; the robot reference point is in $(x, \cdot)$ (the $y$ coordinate is irrelevant) and its rotation is $\omega$; there is a single sliding contact $\left(r_{i}, \phi_{i}\right)$.

We assume that the description of the feasible region for the contacts is provided as a function $v:[0,+\infty] \times[-\pi, \pi[\mapsto\{0,1\}$. A position $(r, \phi)$, expressed in polar coordinates, is in the feasible region if and only if $v(r, \phi)=1$.

A sliding contact $\left(r_{i}, \phi_{i}\right)$ is over a positive strip if and only if $\exists k \in \mathbb{N}$ : $2 k(w+v) \leq x+r_{i} \cos \left(\phi_{i}+\omega\right) \leq(2 k+1)(w+v)-v$. The same condition can also be expressed in terms of remainder of the division, which allows to remove $k:\left(x+r_{i} \cos \left(\phi_{i}+\omega\right)\right) \bmod (2 w+2 v) \leq w$. We define a function $f_{c}^{+}$which is 1 if the condition is met and 0 otherwise:

$$
f_{c}^{+}(r, \phi, x, \omega)= \begin{cases}1 & \text { if }\left(x+r_{i} \cos \left(\phi_{i}+\omega\right)\right) \bmod (2 w+2 v) \leq w \\ 0 & \text { otherwise }\end{cases}
$$

Similarly, we define the function $f_{c}^{-}$for negative strips:

$$
f_{c}^{-}(r, \phi, x, \omega)= \begin{cases}1 & \text { if } w+v \leq\left(x+r_{i} \cos \left(\phi_{i}+\omega\right)\right) \bmod (2 w+2 v) \leq 2 w+v \\ 0 & \text { otherwise }\end{cases}
$$


We can hence write the powered condition of a robot in position $x$ and rotation $\omega$ as:

$$
\begin{aligned}
& f^{+}(A, x, \omega)=\sum_{i=1}^{i=n} f_{c}^{+}\left(r_{i}, \phi_{i}, x, \omega\right) v\left(r_{i}, \phi_{i}\right) \geq 1 \\
& f^{-}(A, x, \omega)=\sum_{i=1}^{i=n} f_{c}^{-}\left(r_{i}, \phi_{i}, x, \omega\right) v\left(r_{i}, \phi_{i}\right) \geq 1
\end{aligned}
$$

where the factor $v\left(r_{i}, \phi_{i}\right)$ means that only contacts which are in the feasible region can be taken into account. The powered condition can also be written as:

$$
f^{+-}(A, x, \omega)=\min \left(f^{+}(A, x, \omega), f^{-}(A, x, \omega)\right) \geq 1
$$

where $f^{+-}$is the number of powered contacts with the robot in position $x$ and rotation $\omega$. Finally, the condition that the robot is in a powered condition in any position $x$ and rotation $\omega$, i.e., the condition for $A$ being a powered array, can be written as:

$$
f(A)=\min _{\substack{x \in[0,2(w+v)[ \\\omega \in[0,2 \pi[}} f^{+-}(A, x, \omega) \geq 1
$$

The problem may hence be tackled by maximizing $f(A)$. Note that, beyond guaranteeing that an array is powered if $f(A) \geq 1$, the chosen $f$ is such that the larger its value, whose upper bound is $\left\lfloor\frac{n}{2}\right\rfloor$, the better the positions of the contacts of the array, since there are more powered contacts.

\section{$2.2 \quad$ Secondary objectives}

It can be seen that, due to the presence of min and mod operators and of the binary co-domain of the function $v, f$ may be extremely non-smooth. Figure 2 shows how $f^{+-}$varies with the position $x$ (left) and rotation $\omega$ (right) of a robot with 5 sliding contacts equally spaced on a circle of radius $r_{0}=20 \mathrm{~mm}$ centered in the reference point-i.e., $\left(r_{i}, \phi_{i}\right)=\left(r_{0},(i-1) \frac{2 \pi}{5}\right)$ with $i \in\{1, \ldots, 5\}$ - which moves on a powered floor with $w=9 \mathrm{~mm}$ and $v=1 \mathrm{~mm}$ : for this specific contacts array $A, f(A)$ is 0 , which means that there are positions and rotations for which the powered condition is not met.

The non-smoothness of $f$ may negatively affect the effectiveness and efficiency of the search for a powered array. In order to address this problem, we consider a function $\hat{f}$ which is the average value of $f^{+-}$, instead of the min value, as in Equation 6:

$$
\hat{f}(A)=\frac{1}{4 \pi(w+v)} \underset{\substack{x \in[0,2(w+v)[ \\\omega \in[0,2 \pi[}}{ } f^{+-}(A, x, \omega) \mathrm{d} x \mathrm{~d} \omega
$$

Instead of measuring the number of powered contacts in the worst condition as $f, \hat{f}$ measures the average number of powered contacts across all the possible 

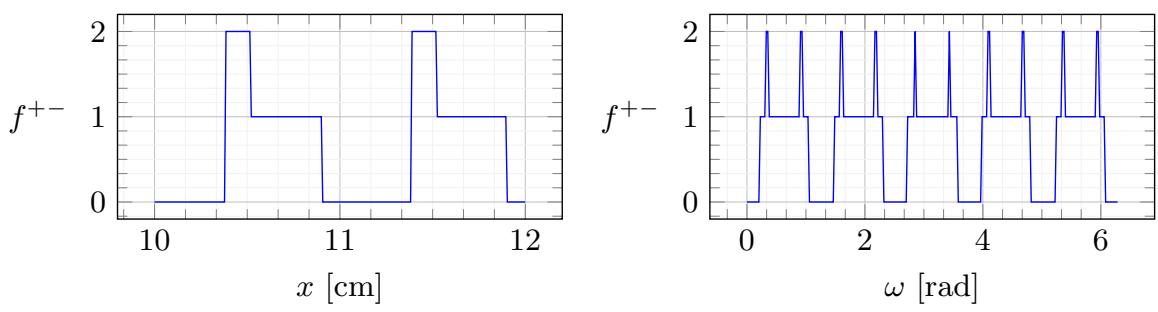

Fig. 2. Values of the function $f^{+-}(A, x, \omega)$ for a simple array of 5 contacts $\left(r_{0},(i-1) \frac{2 \pi}{5}\right)$ (equally spaced on a circle of radius $r_{0}=20 \mathrm{~mm}$ centered in the robot reference point) for different positions $(x$, left) and rotations $(\omega$, right) of the robot, with $w=9 \mathrm{~mm}$ and $v=1 \mathrm{~mm}$.

conditions. It is worth to note, however, that while $f \geq 1$ implies the the robot is always (i.e., for any $x$ and $\omega$ ) in a powered condition, $\hat{f} \geq 1$ does not. That is, $\hat{f}(A) \geq 1$ does not guarantee that an array $A$ is a powered array. In the next sections, we will show how we used $f$ and $\hat{f}$ together to drive the search for a powered array.

The function $f$ does not explicitly take into account the relative positions of the sliding contacts. In particular, it does not penalize designs of the contacts array where the sliding contacts are too close to each other: those design might be harder to be realized in practice. To take into account this aspect, we introduce a third objective function $d$ which measures the average distance of the contacts to their closest contact:

$$
d(A)=\frac{1}{n} \sum_{i \in V} \min _{j \in V, j \neq i} \sqrt{r_{i}^{2}+r_{j}^{2}-2 r_{i} r_{j} \cos \left(\phi_{i}-\phi_{j}\right)}
$$

where $V=\left\{1 \leq i \leq n: v\left(r_{i}, \phi_{i}\right)=1\right\}$ is the set of the indexes of the contacts which are in the feasible region-i.e., as for $f$ and $\hat{f}$, sliding contacts which are not in the feasible region are not taken into account by $d$.

\subsection{Optimization}

We resort to Differential Evolution (DE) [10] for solving the problem of the design of contacts array. DE is an EA which can be used for optimization in continuous (real-valued) search space both for single- and multi-objective problems and hence fits the scenario of this study. There exist many variants of DE which are commonly identified with the $\mathrm{DE} / \mathrm{a} / \mathrm{b} / \mathrm{c}$ naming scheme [11]: in this work, we used the DE/rand/1, i.e., a variant without the crossover operator which selects the individual to be mutated randomly.

In brief, DE/rand/1 evolves a population of $n_{\text {pop }}$ vectors $\boldsymbol{x} \in R^{n_{s}}$ as shown in Algorithm 1. The population is first initialized (lines 1-6) by randomly setting solutions elements. Then, the following steps are repeated until a termination criterion is met: (i) three different individuals $\boldsymbol{x}, \boldsymbol{y}, \boldsymbol{z}$ are randomly selected in 
the population (with uniform probability); (ii) a new solution $\boldsymbol{x}^{\prime}$ is built in which each element $x_{j}^{\prime}$ is set either to $x_{j}$ (with a probability $1-c_{r}$ ) or to a linear combination $x_{j}+d_{w}\left(y_{j}-z_{j}\right)$ of the corresponding elements of the selected individuals (with a probability $c_{r}$ ); (iii) if $\boldsymbol{x}^{\prime}$ is fitter than $\boldsymbol{x}$, then $\boldsymbol{x}^{\prime}$ replaces $\boldsymbol{x}$ in the population, otherwise it is discarded. A usual termination criterion consists in having performed $n_{\mathrm{ev}}$ iterations, which correspond to $n_{\mathrm{ev}}$ fitness evaluations.

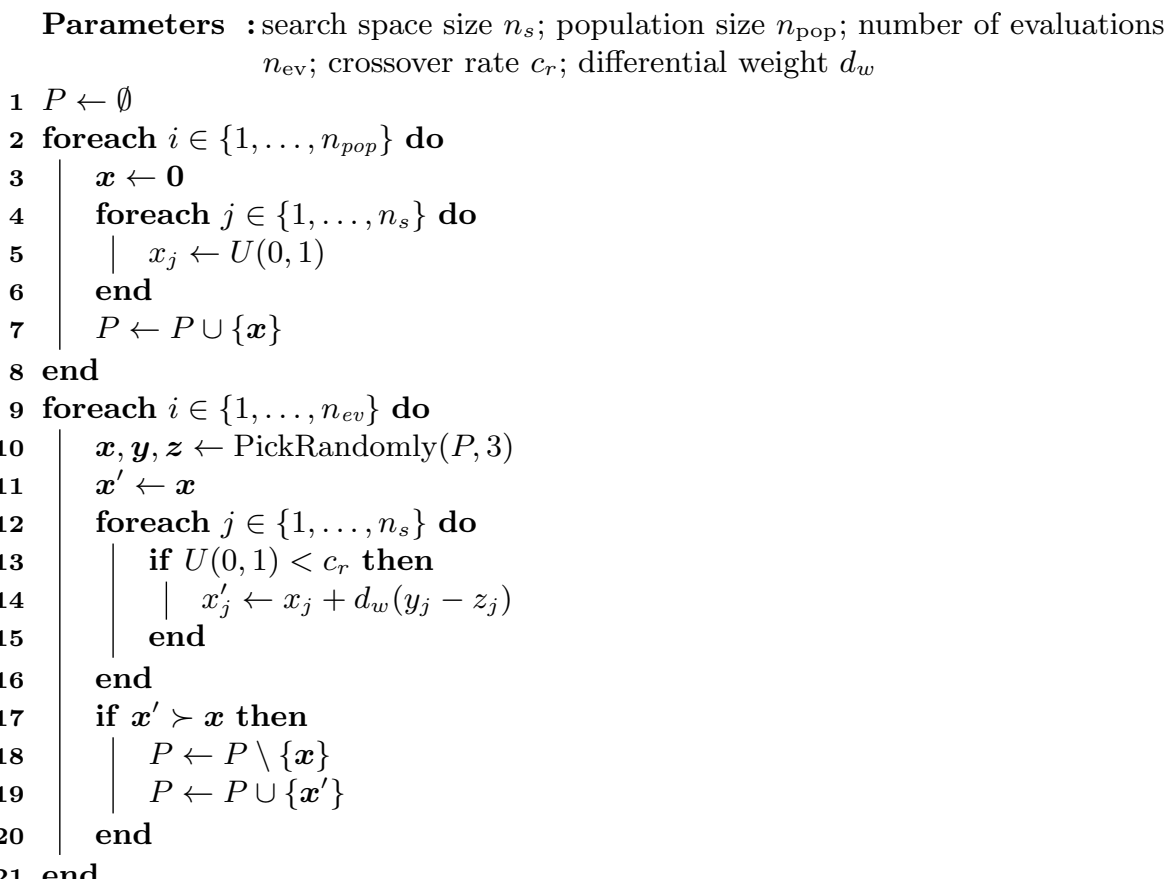

Algorithm 1: Differential Evolution in the DE/rand/1 variant.

In a multi-objective problem, the comparison between two individuals $\boldsymbol{x}, \boldsymbol{x}^{\prime}$ for determining the fittest one can be done in several ways. In this work, we use lexicographical ordering. Let $\boldsymbol{f}(\boldsymbol{x}) \in \mathbb{R}^{m}$ be the $m$-dimensional fitness vector of the solution $\boldsymbol{x}$ and let assume, without loss of generality, that we want to maximize fitness objectives. We say that $\boldsymbol{x}^{\prime}$ is fitter than $\boldsymbol{x}$, denoted by $\boldsymbol{x}^{\prime} \succ \boldsymbol{x}$, if and only if $\exists k \leq m:\left(\forall i<k: f_{i}\left(\boldsymbol{x}^{\prime}\right)=f_{i}(\boldsymbol{x})\right) \wedge\left(f_{k}\left(\boldsymbol{x}^{\prime}\right)>f_{k}(\boldsymbol{x})\right)$.

In this study, we explored different options for driving the search, i.e., different $\boldsymbol{f}$ based on $f, \hat{f}$, and $d$ functions. Concerning $f$ and $\hat{f}$, the actual global minimum or average value of $f^{+-}(A, x, \omega)$ for $x \in[0,2(w+v)[$ and $\omega \in[0,2 \pi[$ (see Equations 6 and 7) cannot, in general, be obtained. Instead, a numerical approximation can be computed. In this work, we compute the values of $f^{+-}$ for the $n_{\text {points }}^{2}(x, \omega)$ pairs resulting from evenly sampling the two corresponding domains and take the minimum (for $f$ ) or average (for $\hat{f}$ ) of those values. 


\section{Experiments and results}

We aimed at gaining insights about our proposed solution from the point of view of the evolutionary optimization and of the considered application. In particular, we aimed at answering the following research questions:

RQ1: are the three objectives $f, \hat{f}$, and $d$, possibly combined, suitable for driving the search for a powered array?

RQ2: how do the main problem parameters (maximum number $n$ of contacts and strips width $w$ ) affect the search for a contact array which always meets the powered condition?

\subsection{Robots}

We applied our proposed method for the automatic design of contacts arrays to a set of problems inspired by the geometry of three real robotic platforms: Thymio $\mathrm{II}^{1}, \mathrm{mBot}^{2}$, and Elisa- $3^{3}$. The three robots, shown in Figure 3, have similar size and were designed to ease learning and experimenting, in particular for children. They are also suitable (and have been actually used [12-14]) for experimenting with Evolutionary Robotics [6].

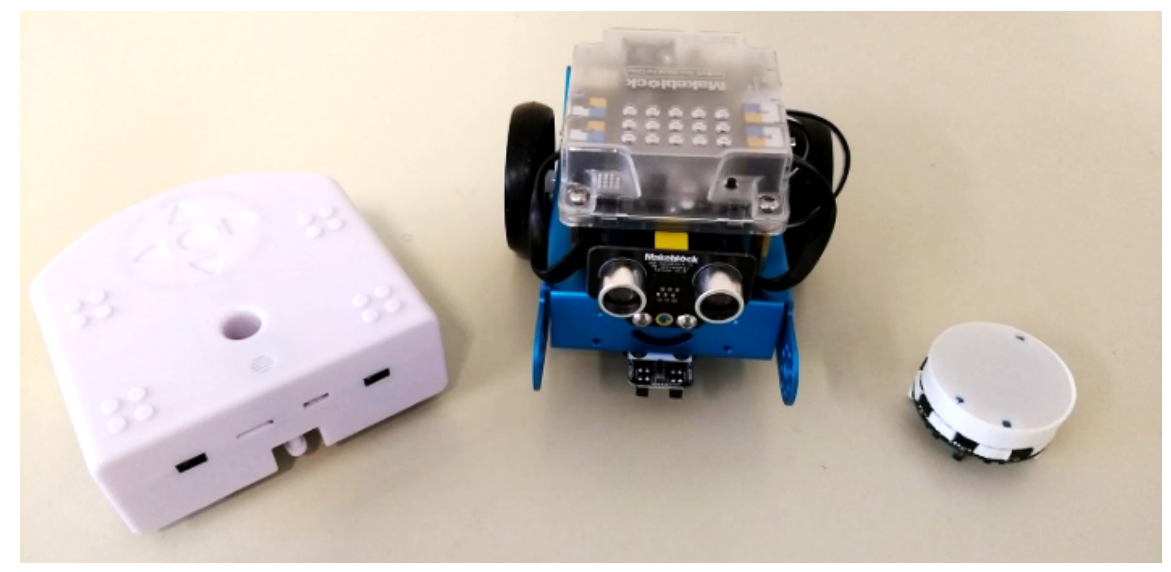

Fig. 3. Thymio II (left), mBot (center), and Elisa-3 (right) robots.

The feasible regions for the three robots are determined by the respective shapes and positions of the wheels and other parts which are designed to be in

\footnotetext{
${ }^{1}$ https://www.thymio.org/en:thymio

${ }^{2}$ https://www.makeblock.com/steam-kits/mbot

3 http://www.gctronic.com/doc/index.php/Elisa-3
} 
contact with the floor:

$$
\begin{aligned}
& v(r, \phi)=\left\{\begin{array}{ll}
1 & \text { if }-50 \mathrm{~mm} \leq r \sin \phi \leq 0 \wedge|r \cos \phi| \leq 75 \mathrm{~mm} \\
1 & 0 \leq r \sin \phi \leq 30 \mathrm{~mm} \wedge|r \cos \phi| \leq 110 \mathrm{~mm} \\
0 & \text { otherwise }
\end{array}\right. \text { for Thymio II } \\
& v(r, \phi)= \begin{cases}1 & \text { if }|r \cos \phi| \leq 45 \mathrm{~mm} \wedge|r \sin \phi| \leq 45 \mathrm{~mm} \\
0 & \text { otherwise }\end{cases} \\
& v(r, \phi)= \begin{cases}1 & \text { if } 25 \mathrm{~mm} \leq r \leq 30 \mathrm{~mm} \\
0 & \text { otherwise }\end{cases}
\end{aligned}
$$

A graphical representation of the feasible regions is shown in Figure 4.

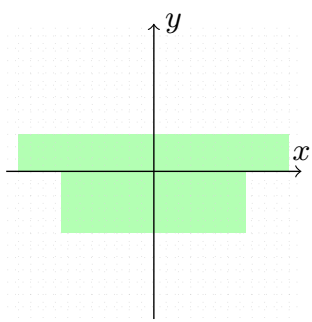

(a) Thymio II.

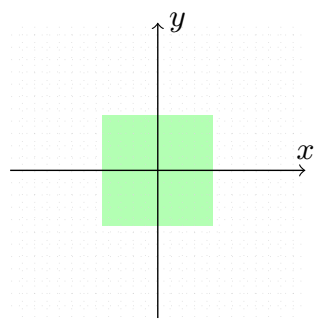

(b) mBot.

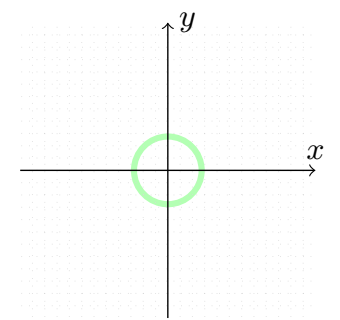

(c) Elisa-3.

Fig. 4. Graphical representation of the feasible regions for the three robots, using Cartesian coordinates $x, y$, rather than polar coordinates $r, \phi$ : regions whose points are such that $v(r, \phi)=1$, with $r=\sqrt{x^{2}+y^{2}}$ and $\phi=\tan ^{-1} \frac{y}{x}$, are plotted in green. The scale is the same for the three plots.

\subsection{Parameters and procedure}

Setting appropriate values for the salient DE parameters (population size $n_{\text {pop }}$, crossover rate $c_{r}$, and differential weight $d_{w}$ ) is not trivial [15]. Based on some preliminary experiments and previous knowledge, we set $n_{\text {pop }}=100, c_{r}=0.8$, and $d_{w}=0.5$. We verified that small variations of those parameters do not substantially alter the qualitative findings of the experimental evaluation.

Concerning the representation, we encoded the position of the contacts in the array by means of a function $e:\left(\mathbb{R}^{+} \times[0,2 \pi]\right)^{n} \mapsto \mathbb{R}^{2 n}$ as follows:

$$
\boldsymbol{x}=e(A)=\left(\frac{r_{1}}{r^{*}}, \frac{\phi_{1}}{2 \pi}, \ldots, \frac{r_{n}}{r^{*}}, \frac{\phi_{n}}{2 \pi}\right)
$$


where $n$ is the maximum number of sliding contacts and $r^{*}$ is the maximum value of $r$ for which $v(r, \phi)=1$, i.e., the distance of the farthest point of the feasible region from the reference point. This way, the initialization procedure of the population, which sets the elements of $\boldsymbol{x}$ by sampling $U(0,1)$ (see Algorithm 1) is appropriate for the domains of $r_{i}, \phi_{i}$.

Concerning the fitness $f$ of the individuals, we experimented with three different options:

$$
\begin{aligned}
\boldsymbol{f}(\boldsymbol{x})=\boldsymbol{f}_{\mathrm{M}}(\boldsymbol{x}) & =\left(f\left(e^{-1}(\boldsymbol{x})\right)\right) \\
\boldsymbol{f}(\boldsymbol{x})=\boldsymbol{f}_{\mathrm{MD}}(\boldsymbol{x}) & =\left(f\left(e^{-1}(\boldsymbol{x})\right), d\left(e^{-1}(\boldsymbol{x})\right)\right) \\
\boldsymbol{f}(\boldsymbol{x})=\boldsymbol{f}_{\mathrm{MAD}}(\boldsymbol{x}) & =\left(f\left(e^{-1}(\boldsymbol{x})\right), \hat{f}\left(e^{-1}(\boldsymbol{x})\right), d\left(e^{-1}(\boldsymbol{x})\right)\right)
\end{aligned}
$$

where $e^{-1}$ is the inverse of the function $e$. The M option corresponds to driving the search using only the function $f$, i.e., a single-objective optimization, whereas MD and MAD use also $d$ and $\hat{f}$.

For each experiment, we performed 30 independent runs by varying the initial random seed: we present mean $\mu$ and standard deviation $\sigma$ computed across the runs for the salient indexes. We set $n_{\mathrm{ev}}=10000$ and $n_{\text {points }}=100$. We performed the experiments on a machine equipped with an Intel(R) Core(TM) i5-3470 CPU at $3.20 \mathrm{GHz}$ with $8 \mathrm{~GB}$ of RAM: one run took, on average, $\approx 80 \mathrm{~s}$ to be performed.

\subsection{Results and discussion}

RQ1: fitness variant. Table 1 shows the value of $f$ obtained at the end of the evolutionary search for the three robots with the three fitness variants with $w=25 \mathrm{~mm}, v=3 \mathrm{~mm}$, and $n=10$.

\begin{tabular}{|c|c|c|c|c|c|c|}
\hline \multirow[b]{2}{*}{ Robot } & \multicolumn{2}{|c|}{ MAD } & \multicolumn{2}{|c|}{ MD } & \multicolumn{2}{|c|}{ M } \\
\hline & $\mu$ & $\sigma$ & $\mu$ & $\sigma$ & $\mu$ & $\sigma$ \\
\hline Elisa-3 & 2 & 0 & 1.48 & 0.51 & 1.760 & 0.44 \\
\hline mBot & 2.03 & 0.19 & & 0 & 2 & 0 \\
\hline Thymio II & 2 & 0 & 0.24 & 0.44 & $0.83 c$ & 0.38 \\
\hline
\end{tabular}

Table 1. Final value of $f$ for the three robots and the three search variants with $w=25 \mathrm{~mm}, v=3 \mathrm{~mm}$, and $n=10$.

It can be seen from the figures in Table 1 that there are differences among the robots and among the fitness variants. With the simplest fitness variant $\mathrm{M}$, our proposed solution is able to obtain, on average, a final $f$ of 1.76 for Elisa-3, 2 for mBot, and 0.83 for Thymio II. We recall that an array of contacts is a powered array (i.e., with that array the robot is in a powered condition in any 
position and rotation) when $f \geq 1$ (see Equation 6 ): hence, our proposed solution with the $\mathrm{M}$ variant is able to design a powered array for two of the three robots. For the Thymio II robot, the $\mathrm{M}$ variant is not able to design a powered array, neither is the MD variant. With the MAD variant, instead, our solution always designs a powered array, i.e., for each robot (note also the $\sigma$ values in Table 1 ). We hypothesize that the reason for which the search struggles for the Thymio II robot is because its feasible region has a more complex shape than those of the other robots.

By analyzing the raw results, we verified that MAD outperforms M and MD because, when the evolution stagnates on a value of $f$, improvements in the value of $\hat{f}$ allow to gradually improve the array design. In other words, thanks to its better smoothness, $\hat{f}$ can drive the search when $f$ cannot. The $d$ function-which, we recall, measures the average distance among contacts in the feasible region (see Equation 8)-does not seem to be able to drive the search better than $f$ : the MD variant, in facts, obtains worse results than the $\mathrm{M}$ variant. This difference between $\hat{f}$ and $d$ is not surprising, however. On one hand, we introduced $\hat{f}$ with the precise goal of mitigating the poor smoothness of $f$, hence as a helper for driving the search together with $f$. On the other hand, $d$ represents a different goal, i.e., designing a contact array which, by avoiding contacts which are too close, is easier to be realized. From a different point of view, $f$ and $\hat{f}$ are not competitive objectives, whereas $f$ and $d$ are: in the extreme case, $d$ pushes the search towards removing contacts (i.e., moving them away from the feasible region), because this increases the average distance; a counter-effect of removing a contact is that $f$ may become lower. Note, however, that since we use lexicographical ordering of objectives for designs comparison (see Section 2.3), a change in a design which negatively affects $f$ will never be kept during the search, regardless of the fact that it improves $d$.

Figure 5 shows the average values of $f$ during the search for the three robots (plots) and the three variants (line colors).

Elisa-3

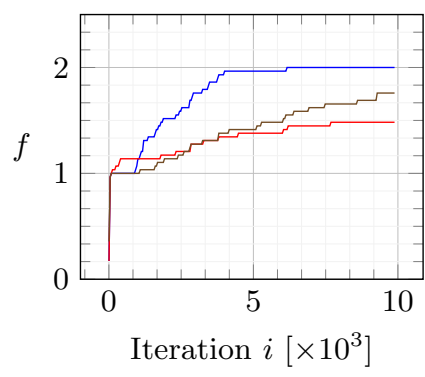

mBot

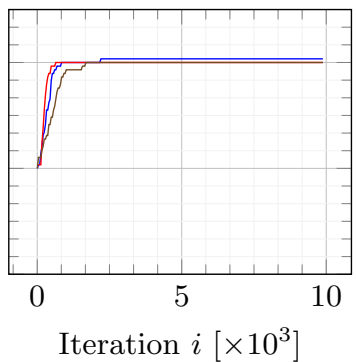

Thymio II

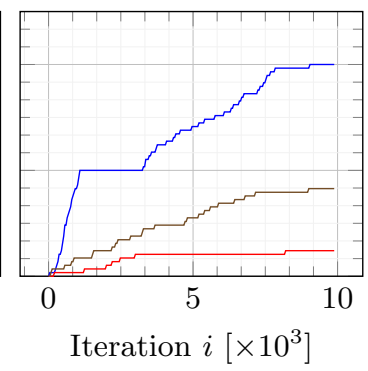

Fig. 5. Value of $f$ during the evolution for the three robots (plots) with the three fitness variants (line color) with $w=25 \mathrm{~mm}, v=3 \mathrm{~mm}$, and $n=10$. 
The figure makes apparent the fact that, for two on three robots (Elisa-3 and Thymio II), the MAD variant is more effective and more efficient than $\mathrm{M}$ and $\mathrm{MD}$; that is, the final value of $f$ is greater and it is reached in fewer iterations. For the mBot the differences among M, MD, and MAD variants are negligible: we motivate this finding with the fact that the feasible region for $\mathrm{mBot}$ is easier than for Elisa-3 and Thymio II. In particular, it is more regular than the one of the latter and much larger than the one of the former.

RQ2: problem parameters. We considered only the best performing variant of our proposed solution (MAD) and performed a set of experiments by varying the value for $w \in\{15,20,25,30,35\} \mathrm{mm}$ and for $n \in\{4,5,6,8,10,12,15\}$, i.e., the width of the conductive strips and the maximum number of contacts in the array, respectively. Figure 6 presents the average final values of the three objectives $f, \hat{f}, d$ for each robot (color line) and each one of the two parameters ( $w$ and $n$, row of plots) - as for the previous experimental campaign, we performed 30 runs for each combination of parameters.
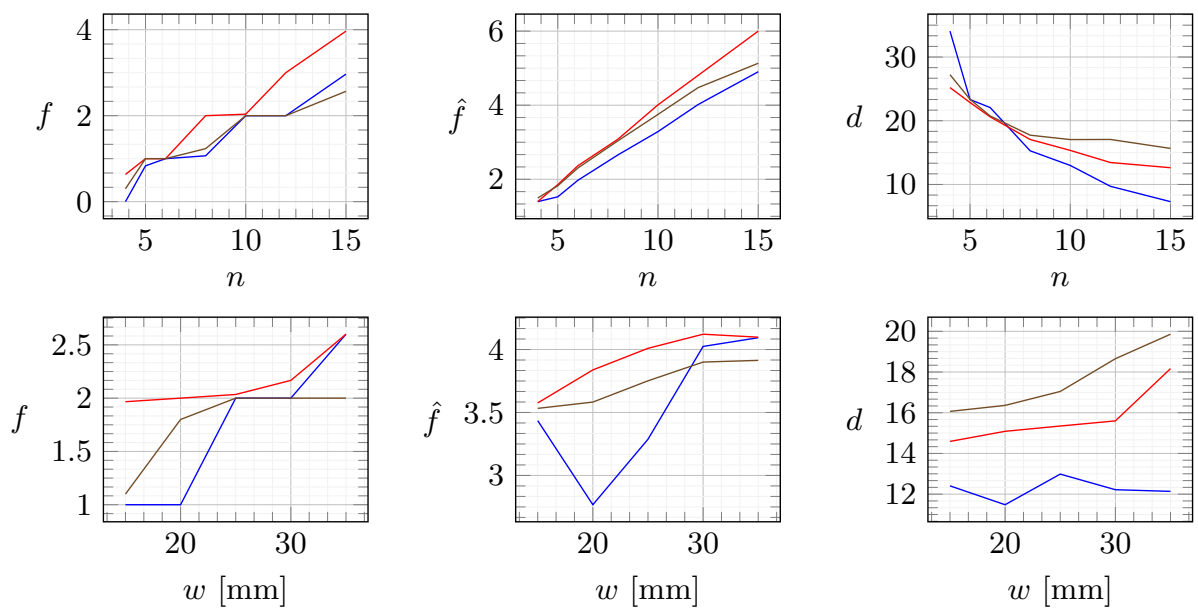

Elisa-3 - mBot — Thymio II

Fig. 6. Final values, averaged across the 30 runs, for the salient functions $f, \hat{f}$, and $d$ for different values for the maximum number $n$ of contacts (top row of plots, with $w=25 \mathrm{~mm}$ ) and different values for the width $w$ of the conductive strips (bottom row of plots, with $n=10$ ) and with $v=3 \mathrm{~mm}$.

The dependency between the three objectives and $n$, visible in Figure 6 , looks as expected. The greater the (maximum) number of contacts $n$, the greater the values of $f$ and $\hat{f}$ and the smaller the value of $d$. Concerning $f, \hat{f}$, we observe that increasing $n$ results, obviously, in more contacts in the array, and hence an opportunity for more powered contacts. Concerning the average distance among 
contacts $d$, the finding is sound: if more contacts are spread on the same feasible region, they are in general closer. Interestingly, the two leftmost plots in the top row of plots of Figure 6 also constitute an evidence of the better smoothness of $\hat{f}$ with respect to $f$ : the lines for the former are essentially straight lines, whereas in the lines for $f$ sort of steps can be spotted in the plot.

From the bottom row of plots of Figure 6 it can be seen that the relation between $w$ and the objectives is more complex than the one between $n$ and the objectives. For example, the final $\hat{f}$ for the Elisa-3 robot has a minimum for $w=20 \mathrm{~mm}$, i.e., greater $\hat{f}$ values are obtained for both $w=15 \mathrm{~mm}$ and $w=25 \mathrm{~mm}$. More in general, it can be seen from these three plots that the relation between the objective and the parameter $w$ varies across objectives and across robots. From a very high level point of view, these plots suggest that the larger the conductive strips, the better: we motivate this finding by observing that increasing $w$ while keeping $v$ (width of non-conductive strips) constant corresponds, basically, to increasing the ratio of the floor area which is actually powered. Concerning the average contacts distance $d$, Figure 6 suggests that the wider the conductive strips, the larger the distance among contacts.

\section{Conclusions and future work}

In this paper, we focused on powered floor systems for delivering power to mobile robots. We considered the problem of automatically finding the positions of the sliding contacts which guarantee that the robot actually receives power from the floor in any position and rotation, the positions being constrained in a given feasible region defined depending on the robot shape and equipment. We introduced a formal formulation for the problem including three objectives to be maximized and constraint to be respected. We tackled the resulting multiobjective optimization problem with Differential Evolution (DE).

We experimentally verified that our proposed solution was indeed able to design effective arrays of sliding contacts for three real robots (Thymio II, mBot, Elisa-3). We investigated about the ability of the three objectives to drive the search with DE. We also experimentally explored the impact of the two most important problem parameters (the width of the conductive strips and the maximum number of sliding contacts) on the effectiveness of the contact arrays designed automatically with DE.

The experimental results suggest that our solution may be useful in practice for assisting the design of powered floor systems. Nevertheless, we think that our work might be extended to take into account finer details of the considered scenario as, for example: (i) a more realistic model for the contact-strip conductivity (which is here binary); (ii) the preference for arrays where contacts on positive and negative strips are, in general, balanced; (iii) the possibility of imposing symmetries in the array. 


\section{References}

1. Shing, A., Wong, P.: Wear of pantograph collector strips. Proceedings of the Institution of Mechanical Engineers, Part F: Journal of Rail and Rapid Transit 222(2), 169-176 (2008)

2. Pastena, L.: A catenary-free electrification for urban transport: An overview of the tramwave system. IEEE Electrification Magazine 2(3), 16-21 (2014)

3. Wang, J., Hu, M., Cai, C., Lin, Z., Li, L., Fang, Z.: Optimization design of wireless charging system for autonomous robots based on magnetic resonance coupling. AIP Advances 8(5), 055004 (2018)

4. Yang, M., Yang, G., Li, E., Liang, Z., Lin, H.: Modeling and analysis of wireless power transmission system for inspection robot. In: Industrial Electronics (ISIE), 2013 IEEE International Symposium on. pp. 1-5. IEEE (2013)

5. Brambilla, M., Ferrante, E., Birattari, M., Dorigo, M.: Swarm robotics: a review from the swarm engineering perspective. Swarm Intelligence 7(1), 1-41 (2013)

6. Nolfi, S., Bongard, J., Husbands, P., Floreano, D.: Evolutionary robotics. In: Springer Handbook of Robotics, pp. 2035-2068. Springer (2016)

7. Watson, R.A., Ficici, S.G., Pollack, J.B.: Embodied evolution: Distributing an evolutionary algorithm in a population of robots. Robotics and Autonomous Systems 39(1), 1-18 (2002)

8. Klingner, J., Kanakia, A., Farrow, N., Reishus, D., Correll, N.: A stick-slip omnidirectional powertrain for low-cost swarm robotics: Mechanism, calibration, and control. In: Intelligent Robots and Systems (IROS 2014), 2014 IEEE/RSJ International Conference on. pp. 846-851. IEEE (2014)

9. Sloane, N.J., Wyner, A.D.: Claude Elwood Shannon: Collected Papers. IEEE press (1993)

10. Storn, R., Price, K.: Differential evolution-a simple and efficient heuristic for global optimization over continuous spaces. Journal of global optimization 11(4), 341-359 (1997)

11. Das, S., Suganthan, P.N.: Differential evolution: a survey of the state-of-the-art. IEEE transactions on evolutionary computation 15(1), 4-31 (2011)

12. Heinerman, J., Rango, M., Eiben, A.: Evolution, individual learning, and social learning in a swarm of real robots. In: Computational Intelligence, 2015 IEEE Symposium Series on. pp. 1055-1062. IEEE (2015)

13. Heinerman, J., Zonta, A., Haasdijk, E., Eiben, A.E.: On-line evolution of foraging behaviour in a population of real robots. In: European Conference on the Applications of Evolutionary Computation. pp. 198-212. Springer (2016)

14. Silva, F., Correia, L., Christensen, A.L.: Evolutionary online behaviour learning and adaptation in real robots. Royal Society open science 4(7), 160938 (2017)

15. Brest, J., Greiner, S., Boskovic, B., Mernik, M., Zumer, V.: Self-adapting control parameters in differential evolution: A comparative study on numerical benchmark problems. IEEE transactions on evolutionary computation 10(6), 646-657 (2006) 